Research Article

\title{
Free Vibration Analysis for Cracked FGM Beams by Means of a Continuous Beam Model
}

\author{
E Chuan Yang, ${ }^{1,2}$ Xiang Zhao, ${ }^{1}$ and Ying Hui Li ${ }^{1}$ \\ ${ }^{1}$ School of Mechanics and Engineering, Southwest Jiaotong University, Chengdu 610031, China \\ ${ }^{2}$ School of Mechanical of Engineering, Chongqing University of Technology, Chongqing 400054, China
}

Correspondence should be addressed to Ying Hui Li; yhli2007@sina.com

Received 10 December 2014; Accepted 26 January 2015

Academic Editor: Yumin He

Copyright (c) 2015 E Chuan Yang et al. This is an open access article distributed under the Creative Commons Attribution License, which permits unrestricted use, distribution, and reproduction in any medium, provided the original work is properly cited.

Based on Euler-Bernoulli beam theory and a continuous stiffness beam model, the free vibration of rectangular-section beams made of functionally graded materials (FGMs) containing open edge cracks is studied. Assuming the material gradients follow exponential distribution along beam thickness direction, the conversion relation between the vibration governing equations of a FGM beam and that of an isotropic homogenous beam is deduced. A continuous function is used to characterize the bending stiffness of an edge cracked FGM beam. Thus, the cracked FGM beam is treated as an intact beam with continuously varying bending stiffness along its longitudinal direction. The characteristic equations of beams with different boundary conditions are obtained by transfer matrix method. To verify the validity of the proposed method, natural frequencies for intact and cracked FGM beams are calculated and compared with those obtained by three-dimensional finite element method (3D FEM) and available data in the literature. After that, further discussions are carried out to analyze the influences of crack depth, crack location, material property, and slenderness ratio on the natural frequencies of the cracked FGM beams.

\section{Introduction}

Functionally graded materials (FGMs) are composite materials made from at least two components that follow a certain material gradient distribution. Because the characteristics of FGMs change continuously in the gradient direction, their fatigue properties are improved [1]. Extensive research works on FGM structures have been undertaken because of the wide range of applications of FGMs [2-9]. Benatta et al. [2] investigated the static bending problem of composite beams composed of various fibers using analytical methods. Ding et al. [3] deduced the stress function of the plane problem for anisotropic FGM beams. Ben-Oumrane et al. [4] studied the static bending of ceramic-metallic FGM beams and quantitatively analyzed the effects of material gradient on the deformation and stress of such beams using the numerical method. Li et al. [5] evaluated the 3D analytical solutions of functionally graded magnetoelectroelastic circular plates subjected to uniform load using polynomial expansion method. Li et al. [6] further obtained the 3D elasticity solutions of functionally graded circular plates by reducing the solutions of an FGM plate to those of a homogeneous plate. Li et al. [7,8], assuming that the material gradients follow power functions along thickness direction of FGM beams, obtained the elastic solutions of buckling and bending problems of FGM beams and determined the conversion relations between the solutions of FGM beams and those of homogeneous beams. Arnaldo and Mazzei [9] studied the natural frequencies of rotating FGM beams using assumed mode method and discussed the effects of material properties on natural frequencies of the beams.

The presence of cracks can lead to structural failures and sometimes results in disasters. Moreover, a crack in a structure influences its dynamic behaviors and such an influence may be employed in the crack identification of a damaged structure to predict an incipient structural failure [10]. Therefore, the recognition of this influence is very important due to its great values of practical application. There are a number of theoretical crack-modeling techniques in the literature, which can be basically grouped into two 
categories: lumped flexibility models [10-15] and continuous stiffness models [16-22]. For the lumped flexibility models, a crack is represented by a rotational spring. The flexibility matrix of the rotational spring is generally determined by the strain energy release rate and the stress intensity factors (SIFs). Dimarogonas [10], based on Euler-Bernoulli beam theory, introduced the initial local flexibility model in which a crack in a beam is represented by a linear rotational springs. Anifantis and Dimarogonas [11], ignoring the torsion effects, came up with a $5 \times 5$ local flexibility matrix of the rotational springs. Papadopoulos and Dimarogonas [12] raised a complete $6 \times 6$ local flexibility matrix based on fracture mechanics theories. In recent years, many research works have been done to study the cracked beams by means of the lumped flexibility models. For example, Xiang et al. $[13,14]$ investigated the natural frequencies and identification problems of cracked shafts; a lumped flexibility model and the wavelet theories are employed to construct the rotating Rayleigh-Euler and Rayleigh-Timoshenko beam elements. Li et al. [15], with the cracks modeled by the linear rotational springs, studied the dynamic responses of cracked Timoshenko beams subjected to transverse concentrated forces. For the continuous stiffness models, researchers attempt to describe the vibration of cracked beams with unified equations in which the information of crack location and crack depth is involved. Using the Hu-Washizu variation principle, Christides and Barr [16], assuming that the stress near a crack follows exponential distribution, completed the 1D integration of stress, strain, and displacement in the edge cracked beam. Subsequently, Christides and Barr [17] expanded their study to the torsion vibration of beams and came up with the corresponding governing equation for the beams containing multiple cracks. Shen and Pierre [18] verified the validity of Christides and Barr's theories with $2 \mathrm{D}$ finite element method. Chondros et al. [19], not limited by the stress assumption in [16], developed the continuous beam models. Based on the energy distribution near the cracktips, Swamidas et al. [20] established continuous bending and shearing stiffness models for the cracked beams. Chomette et al. [21], by means of the continuous beam models, proposed a new method to detect the small cracks in beam structures.

Whatever method is used to model the cracked beams, the models do involve the fracture mechanics theories and stress intensity factors (SIFs). Delale and Erdogan [22-25] performed a lot of detailed researches on the SIFs in nonhomogeneous structures and came up with many important conclusions. They researched the plane problem in infinite plates and drew the conclusion that the effect of Poisson's ratio on the SIFs is very limited [22]. They also investigated the stress distribution near the crack-tip of the FGM plates containing open edge cracks and tabulated the dimensionless SIFs of the cracks of different crack depths and different material gradients [25]. Chan et al. [26] presented an integral equation formulation based on displacement method, which was used to deal with the antiplane problem of FGM plates containing the tearing cracks.

While numerous studies have been performed to investigate the influences of the cracks on the dynamic behaviors of isotropic homogenous beams, there are also some research works focusing on the edge cracked FGM beams which are concerned in this paper. The following presents a literature review related to FGM beams with open edge cracks. Yang and Chen [27] studied the free vibration of cracked FGM beams based on Euler-Bernoulli beam theory and the rotational spring model. The influences of crack location, material properties, and slenderness ratio on the natural frequency of cracked FGM beams were discussed. Ke et al. [28] improved the method described in [27] for cracked Timoshenko FGM beams. Yu and Chu [29] proposed a p-version finite element method to determine the natural frequencies of cracked FGM beams and dealt with the crack identification of the slender cantilever FGM beams containing open edge cracks. Based on Euler-Bernoulli beam theory and the rotational spring model, Aydin [30] used a third-order determinant to analyze the natural frequencies of damaged FGM beams containing arbitrary numbers of cracks.

As is known, natural frequencies are the most commonly used parameters employed in the crack identification of a damaged structure [30]. However, the literature review above shows that a limited amount of research works has been carried out to discuss the vibration problems of edge cracked FGM beams. Moreover, in most reports, rotational springs are used to simulate the cracks in FGM beams. Therefore, the present paper investigates the free vibration of FGM beams containing open edge cracks and proposes a new method to determine the natural frequencies of these beams. More specifically, based on Euler-Bernoulli beam theory, the conversion relation between vibration governing equation of a FGM beam and that of an isotropic homogenous beam is deduced, and a cracked FGM beam is simulated by a continuous beam model. Moreover, the cracked FGM beam is treated as an intact beam with its bending stiffness varying continuously along beam longitudinal direction. Dividing the beam into sufficient number of segments and employing the transfer matrix method, the characteristic equation of the beam is obtained. The validity of the proposed method is verified by the three-dimensional finite element method (3D FEM) and the data in references. Furthermore, the influences of crack depth, crack location, material gradient, and slenderness ratio on the first three natural frequencies of the cracked FGM beams are discussed. The method and the results in this paper can be used for nondestructive testing of beam structures made of FGMs.

\section{Theoretical Models}

2.1. Free Vibration of an Intact FGM Euler-Bernoulli Beam. A FGM Euler-Bernoulli beam, which is considered in this paper, is shown in Figure 1, where $L, h$, and $b$ are, respectively, length, height, and width of the beam, $c$ is the location of the crack and, $a$ is the depth of the crack. The material parameters $E_{1}, \mu_{1}, \rho_{1}$, and $E_{2}, \mu_{2}, \rho_{2}$ denote Young's modulus, shear modulus, and mass density at top and bottom surfaces of the beam, respectively. Young's modulus $E(z)$, shear modulus $\mu(z)$, and mass density $\rho(z)$ are assumed to vary along the thickness direction according to the following expressions:

$$
E(z)=E_{0} e^{\beta z}, \quad \mu(z)=\mu_{0} e^{\beta z}, \quad \rho(z)=\rho_{0} e^{\beta z},
$$




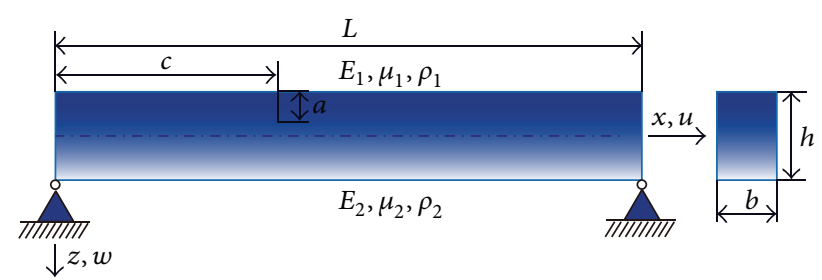

FIgURE 1: A FGM beam with a single open edge crack.

where $\beta$ is a positive or negative constant characterizing the material gradient, $E_{0}, \mu_{0}$, and $\rho_{0}$ are the values of those elastic properties of the middle layer $(y=0)$. Since Poisson's ratio presents a very limited influence on SIFs [22], Poisson's ratio $v$ is taken as a constant.

From Euler-Bernoulli beam theory, the displacement components of an arbitrary point in the beam can be expressed as

$$
u(x, z, t)=u_{0}(x, t)-z \frac{\partial w_{0}}{\partial x}, \quad w(x, z, t)=w_{0}(x, t),
$$

where $\mu(x, z, t)$ and $w(x, z, t)$ are the displacements along $x$ and $z$ axes and $\mu_{0}(x, t)$ and $w_{0}(x, t)$ are the displacements of a point in the middle layer. Accordingly, the following geometric equation can be obtained as

$$
\varepsilon(x, z, t)=\frac{\partial u_{0}}{\partial x}-z \frac{\partial^{2} w_{0}}{\partial x^{2}},
$$

where $\varepsilon(x, z, t)$ is the normal strain of an arbitrary point in the beam. Assuming the normal stress $\sigma$ keeps in a linear relationship with the normal, strain $\varepsilon$, the normal stress $\sigma$ can be written as

$$
\sigma(x, z, t)=E(z) \varepsilon=E(z)\left(\frac{\partial u_{0}}{\partial x}-z \frac{\partial^{2} w_{0}}{\partial x^{2}}\right) .
$$

With (4), the resultant stresses in a cross section of the beam yield the normal force $N$ and bending moment $M$ :

$$
\begin{gathered}
N=\int_{A} \sigma d A=A_{1} \frac{\partial u_{0}}{\partial x}-B_{1} \frac{\partial^{2} w_{0}}{\partial x^{2}}, \\
M=\int_{A} \sigma z d A=B_{1} \frac{\partial u_{0}}{\partial x}-D_{1} \frac{\partial^{2} w_{0}}{\partial x^{2}},
\end{gathered}
$$

where $A_{1}, B_{1}$, and $D_{1}$ are, respectively, defined as

$$
\begin{gathered}
A_{1}=\int_{A} E(z) d A, \quad B_{1}=\int_{A} z E(z) d A, \\
D_{1}=\int_{A} z^{2} E(z) d A .
\end{gathered}
$$

According to Euler-Bernoulli beam theory, the equilibrium equations of the beam are given by [8]

$$
\begin{gathered}
A_{1} \frac{\partial^{2} u_{0}}{\partial x^{2}}-B_{1} \frac{\partial^{3} w_{0}}{\partial x^{3}}=0 \\
B_{1} \frac{\partial^{3} u_{0}}{\partial x^{3}}-D_{1} \frac{\partial^{4} w_{0}}{\partial x^{4}}-\bar{\rho} A \frac{\partial^{2} w_{0}}{\partial t^{2}}+q(x, t)=0,
\end{gathered}
$$

where $\bar{\rho} A=\int_{A} \rho(z) d A$ is the mass per unit length and $q(x, t)$ the transverse distributed load. For free transverse vibration of the beam, substituting (7) into (8) and ignoring the incentive item $q(x, t)$ in (8), the following governing equation can be reduced:

$$
\left(D_{1}-\frac{B_{1}^{2}}{A_{1}}\right) \frac{\partial^{4} w_{0}}{\partial x^{4}}+\bar{\rho} A \frac{\partial^{2} w_{0}}{\partial t^{2}}=0 .
$$

For the situation that the material gradient follows exponential distribution along beam thickness direction, as given in (1), two parameters can be defined as follows:

$$
S_{1}=\sinh \left(\frac{\beta h}{2}\right), \quad S_{2}=\cosh \left(\frac{\beta h}{2}\right),
$$

As thus, $A_{1}, B_{1}, D_{1}$, and $\bar{\rho}$ in (7) and (8) can be represented as

$$
\begin{gathered}
A_{1}=\varphi_{1} A E_{0}, \quad B_{1}=\varphi_{2} b h^{2} E_{0}, \\
D_{1}=\varphi_{3} I E_{0}, \quad \bar{\rho}=\varphi_{4} \rho_{0},
\end{gathered}
$$

where $A$ is the area of cross section and $I$ is the moment of inertia of a rectangular-section beam $\left(I=b h^{3} / 12\right)$ and

$$
\begin{gathered}
\varphi_{1}=\varphi_{4}=\frac{2 S_{1}}{\beta h}, \quad \varphi_{2}=-\frac{2 S_{1}-\beta h S_{2}}{\beta h^{2}}, \\
\varphi_{3}=\frac{6}{\beta^{3} h^{3}}\left(\left(\beta^{2} h^{2}+8\right) S_{1}-4 \beta h S_{2}\right) .
\end{gathered}
$$

Substituting (10)-(12) into (9) yields

$$
E_{0} I c_{E} \frac{\partial^{4} w_{0}}{\partial x^{4}}+\rho_{0} A c_{\rho} \frac{\partial^{2} w_{0}}{\partial t^{2}}=0,
$$

where

$$
c_{E}=\varphi_{3}-\frac{12 \varphi_{2}^{2}}{\varphi_{1}}, \quad c_{\rho}=\varphi_{1} .
$$

From (13), it can be found that, with the nonuniform material coefficients $c_{E}$ and $c_{\rho}$, the governing equation of the FGM beam can be converted to the form which is similar to that of an isotropic homogenous beam. Thus, the solving process of a FGM beam can be degenerated to that of an isotropic homogenous beam.

2.2. Free Vibration of a FGM Beam Containing an Open Edge Crack. Based on fracture mechanics theory, for a rectangular-section beam subjected to bending moments and containing an open edge crack, if $a / h \leq 0.6$, Swamidas et al. [20] proposed the following continuous bending stiffness model:

$$
E I_{c}(x)=\frac{E I}{1+E I R(a, c) /\left(1+((x-c) / k(a) a)^{2}\right)} .
$$


In (15), EI is the bending stiffness of an intact beam and $\operatorname{EIR}(a, c)$ and $k(a)$ are parameters associated with $a$ and $c$ and are given as [20]

$$
\begin{aligned}
& \operatorname{EIR}(a, c) \\
& =\frac{3 \pi[F(a)]^{2} a}{k(a) h[\arctan ((L-c) / k(a) a)+\arctan (c / k(a) a)]}, \\
& k(a)=\frac{3 \pi[F(a)]^{2}(h-a)^{3} a}{\left[h^{3}-(h-a)^{3}\right] h} .
\end{aligned}
$$

In (16) $F(a)$ denotes the normalized SIF. For an open edge crack in a rectangular-section beam subjected to bending moments, it is given as [25]

$$
F(a)=\frac{K_{\mathrm{I}}}{6 M \sqrt{a} / b h^{2}},
$$

where $K_{\mathrm{I}}$ is the SIF of the crack. For open edge cracks in FGM beams, the values of the SIFs for different crack depths (from $a / h=0.1$ to $a / h=0.7$ ) and different material gradient (from $E_{2} / E_{1}=0.1$ to $\left.E_{2} / E_{1}=10\right)$ were tabulated by Erdogan and $\mathrm{Wu}$ [25]. Extracting from those data predicted by Erdogan and $\mathrm{Wu}$ [25], polynomial expressions of the normalized SIFs were obtained by Ke et al. [28] using Lagrange interpolation technique and the detailed expressions can be found in [28].

According to the continuous bending stiffness characterized by (15), a cracked FGM beam can be treated as an intact beam with continuously varying bending stiffness along its longitudinal direction. The governing equation of free vibration of the cracked FGM beam takes the form of a nonuniform beam as

$$
\frac{\partial^{2}}{\partial x^{2}}\left[E_{0} I_{c}(x) c_{E} \frac{\partial^{2} w_{0}}{\partial x^{2}}\right]+\rho_{0} A c_{\rho} \frac{\partial^{2} w_{0}}{\partial t^{2}}=0,
$$

where $c_{E}, c_{\rho}$, and $E_{0} I_{c}(x)$ have been, respectively, defined by (14) and (15). Being different from (13), the analytical solutions of (18) cannot be solved by separation variable method. However, the numerical solutions of (18) can be obtained by means of transfer matrix method. For a beam with varying section parameters along its longitudinal direction, we can divide the beam into $n$ segments. If the length of each segment is short enough, the bending stiffness $(E I)_{i}(i=1,2, \ldots, n)$ and the linear density $(\rho A)_{i}(i=1,2, \ldots, n)$ for each segment can be treated as constants. Then, the governing equation of each segment can also take the following form of a uniform beam:

$$
\begin{gathered}
(E I)_{i} \frac{\partial^{4} w_{0}^{(i)}}{\partial x^{4}}+(\rho A)_{i} \frac{\partial^{2} w_{0}^{(i)}}{\partial t^{2}}=0, \\
x_{i} \leq x \leq x_{i+1}, \quad i=0,1, \ldots, n .
\end{gathered}
$$

In (19), the bending stiffness and the linear density of (i)th segment can be described as

$$
(E I)_{i}=\frac{1}{l_{i}} \int_{x_{i-1}}^{x_{i}} E_{0} I_{c}(x) c_{E} d x, \quad(\rho A)_{i}=\rho_{0} A c_{\rho}
$$

where $l_{i}, x_{i-1}$ and $x_{i}$ denote the length and the coordinates of left end and right end of the (i)th segment.

According to Euler-Bernoulli beam theory, the modal function of the $(i)$ th segment of the beam takes the form of

$$
Y_{i}(x)=H_{i} \sin X_{i}+J_{i} \cos X_{i}+K_{i} \sinh X_{i}+L_{i} \cosh X_{i},
$$

where $X_{i}=\varsigma_{i}\left(x-x_{i-1}\right), x_{i-1} \leq x \leq x_{i}, i=1,2, \ldots, n$, and $H_{i}, J_{i}, K_{i}$ and $L_{i}$ are coefficients to be determined associated with the $(i)$ th beam segment and

$$
\zeta_{i}=\frac{(\rho A)_{i}}{(E I)_{i}} \omega^{2} .
$$

In (22), $\omega$ is the circular frequency of the beam. Similarly, the modal function of the $(i+1)$ th beam segment reads

$$
\begin{aligned}
Y_{i+1}(x)= & H_{i+1} \sin X_{i+1}+J_{i+1} \cos X_{i+1} \\
& +K_{i+1} \sinh X_{i+1}+L_{i+1} \cosh X_{i+1}
\end{aligned}
$$

For the continuity between the $(i)$ th and $(i+1)$ th segment, the transverse deflection, rotation angle, bending moment, and shear force at the common interface of these two segments are required to fulfill the following conditions:

$$
\left[\begin{array}{c}
Y_{i+1}\left(x_{i}\right) \\
Y_{i+1}^{\prime}\left(x_{i}\right) \\
(E I)_{i+1} Y_{i+1}^{\prime \prime}\left(x_{i}\right) \\
\left((E I)_{i+1} Y_{i+1}^{\prime \prime}\left(x_{i}\right)\right)^{\prime}
\end{array}\right]=\left[\begin{array}{c}
Y_{i}\left(x_{i}\right) \\
Y_{i}^{\prime}\left(x_{i}\right) \\
(E I)_{i} Y_{i}^{\prime \prime}\left(x_{i}\right) \\
\left((E I)_{i} Y_{i}^{\prime \prime}\left(x_{i}\right)\right)^{\prime}
\end{array}\right]
$$

Substituting (21)-(23) into (24) yields

$$
\mathbf{M}_{(i+1)}=\mathbf{Z}_{(i)} \mathbf{M}_{(i)},
$$

where

$$
\begin{aligned}
\mathbf{M}_{(i)} & =\left[\begin{array}{llll}
H_{i} & J_{i} & K_{i} & L_{i}
\end{array}\right]^{T}, \\
\mathbf{M}_{(i+1)} & =\left[\begin{array}{llll}
H_{i+1} & J_{i+1} & K_{i+1} & L_{i+1}
\end{array}\right]^{T} .
\end{aligned}
$$

In (25), $\mathbf{Z}_{(i)}$ is the transfer matrix and has the form of

$$
\mathbf{Z}_{(i)}=\left[\begin{array}{cccc}
c_{1} \chi_{i}^{(2)} & -c_{1} \chi_{i}^{(1)} & -c_{2} \chi_{i}^{(4)} & -c_{2} \chi_{i}^{(3)} \\
c_{3} \chi_{i}^{(1)} & c_{3} \chi_{i}^{(2)} & -c_{4} \chi_{i}^{(3)} & -c_{4} \chi_{i}^{(4)} \\
-c_{2} \chi_{i}^{(2)} & c_{2} \chi_{i}^{(1)} & c_{1} \chi_{i}^{(4)} & c_{1} \chi_{i}^{(3)} \\
-c_{4} \chi_{i}^{(1)} & -c_{4} \chi_{i}^{(2)} & c_{3} \chi_{i}^{(3)} & c_{3} \chi_{i}^{(4)}
\end{array}\right]
$$

where $c_{1,2}=\varsigma_{i}(p \pm 1) /\left(2 \varsigma_{i+1}\right), c_{3,4}=\varsigma_{i}(p \pm 1) / 2, p=$ $(E I)_{i} \varsigma_{i}^{2} /\left((E I)_{i+1} \varsigma_{i+1}^{2}\right), \chi_{i}^{(1)}=\sin \varsigma_{i}, \chi_{i}^{(2)}=\cos \varsigma_{i} l_{i}, \chi_{i}^{(3)}=$ $\sinh \varsigma_{i} l_{i}$, and $\chi_{i}^{(4)}=\cosh \varsigma_{i} l_{i}$.

Equation (25) can be viewed as a bridge connecting the physical quantities pertinent to the $(i)$ th and $(i+1)$ th segment. Using (25) repeatedly, we may construct the following relations between the first and the last segments:

$$
\mathbf{M}_{(n)}=\mathbf{Z M}_{(1)},
$$


where $\mathbf{M}_{(1)}$ and $\mathbf{M}_{(n)}$ are vectors, respectively, representing the boundary conditions (transverse deflection, rotation angle, bending moment, and shear force) at both ends of the beam; the transfer matrix $\mathbf{Z}$ is of the following form:

$$
\mathbf{Z}=\mathbf{Z}_{(n-1)} \mathbf{Z}_{(n-2)} \cdots \mathbf{Z}_{(2)} \mathbf{Z}_{(1)}
$$

It should be noted that each factor of the matrix $\mathbf{Z}$ contains the circular frequency. Consequently, (28) is an implicit matrix equation in $\omega$. Once the boundary conditions in $\mathbf{M}_{(1)}$ and $\mathbf{M}_{(n)}$ are given, the circular frequency $\boldsymbol{\omega}$ of the cracked FGM beam can be numerically determined from (28). Then, the modal function can be readily obtained from (21).

2.3. Characteristic Equations for Different Boundary Conditions. In this subsection, the characteristic equations of cracked FGM beams are presented. Three typical boundary conditions (hinged-hinged, clamped-clamped, and clamped-free) are considered case by case.

For a hinged-hinged beam, the boundary conditions are given as

$$
\begin{array}{ccc}
Y_{1}(0)=0, & (E I)_{1} Y_{1}^{\prime \prime}(0)=0 \quad \text { at } x=0, \\
Y_{n}(L)=0, & (E I)_{n} Y_{n}^{\prime \prime}(L)=0 \quad \text { at } x=L .
\end{array}
$$

From (21) and (30a), one can readily have

$$
J_{1}=0, \quad L_{1}=0 .
$$

In view of (21) and (30b), the following matrix equation can be obtained

$$
\Lambda_{1} \mathbf{M}_{(n)}=\mathbf{0}
$$

where $\Lambda_{1}$ takes the form of

$$
\boldsymbol{\Lambda}_{1}=\left[\begin{array}{cc}
\sin \varsigma_{n} l_{n} & -(E I)_{n} \varsigma_{n}^{2} \sin \varsigma_{n} l_{n} \\
\cos \varsigma_{n} l_{n} & -(E I)_{n} \varsigma_{n}^{2} \cos \varsigma_{n} l_{n} \\
\sinh \varsigma_{n} l_{n} & (E I)_{n} \varsigma_{n}^{2} \sinh \varsigma_{n} l_{n} \\
\cosh \varsigma_{n} l_{n} & (E I)_{n} \varsigma_{n}^{2} \cosh \varsigma_{n} l_{n}
\end{array}\right]^{T}
$$

Substitution of (28) into (32) leads to

$$
\Lambda_{1} \mathbf{Z M}_{(1)}=\Gamma_{1} \mathbf{M}_{(1)}=\mathbf{0},
$$

where

$$
\Gamma_{1}=\Lambda_{1} \mathbf{Z}
$$

With (31) and (34), it can be obtained that

$$
\boldsymbol{\Gamma}_{1} \mathbf{M}_{(1)}=\left[\begin{array}{ll}
\Gamma_{11} & \Gamma_{13} \\
\Gamma_{21} & \Gamma_{23}
\end{array}\right]\left[\begin{array}{l}
H_{1} \\
K_{1}
\end{array}\right]=\mathbf{0} .
$$

To make sure there are nonzero solutions for (36), the determinant of the coefficient matrix in (36) has to be equal to zero, so the characteristic equations of the hinged-hinged FGM beam can be finally written as

$$
\operatorname{det}\left[\begin{array}{ll}
\Gamma_{11} & \Gamma_{13} \\
\Gamma_{21} & \Gamma_{23}
\end{array}\right]=0 \text {. }
$$

For clamped-clamped beams the boundary conditions require that

$$
\begin{gathered}
Y_{1}(0)=0, \quad Y_{1}^{\prime}(0)=0, \quad \text { at } x=0, \\
Y_{n}(L)=0, \quad Y_{n}^{\prime}(L)=0, \quad \text { at } x=L .
\end{gathered}
$$

Substituting (21) into (38a) leads to

$$
H_{1}+K_{1}=0, \quad J_{1}+L_{1}=0 .
$$

From (21) and (38b), one has

$$
\Lambda_{2} \mathbf{M}_{(n)}=\mathbf{0}
$$

where $\boldsymbol{\Lambda}_{2}$ has the form of

$$
\Lambda_{2}=\left[\begin{array}{cc}
\sin \varsigma_{n} l_{n} & (E I)_{n} \varsigma_{n} \cos \varsigma_{n} l_{n} \\
\cos \varsigma_{n} l_{n} & -(E I)_{n} \varsigma_{n} \sin \varsigma_{n} l_{n} \\
\sinh \varsigma_{n} l_{n} & (E I)_{n} \varsigma_{n} \cosh \varsigma_{n} l_{n} \\
\cosh \varsigma_{n} l_{n} & (E I)_{n} \varsigma_{n} \sinh \varsigma_{n} l_{n}
\end{array}\right]^{T} .
$$

Substituting (28) into (40) derives

$$
\begin{gathered}
\Lambda_{2} \mathbf{Z M}_{(1)}=\Gamma_{2} \mathbf{M}_{(1)}=\mathbf{0}, \\
\Gamma_{2}=\Lambda_{2} \mathbf{Z} \\
\Gamma_{2} \mathbf{M}_{(1)}=\left[\begin{array}{llll}
\Gamma_{11} & \Gamma_{12} & \Gamma_{13} & \Gamma_{14} \\
\Gamma_{21} & \Gamma_{22} & \Gamma_{23} & \Gamma_{24}
\end{array}\right]\left[\begin{array}{c}
H_{1} \\
J_{1} \\
-H_{1} \\
-J_{1}
\end{array}\right]=\mathbf{0 .}
\end{gathered}
$$

The characteristic equation of the clamped-clamped FGM beam can be determined as

$$
\operatorname{det}\left[\begin{array}{ll}
\Gamma_{11}-\Gamma_{13} & \Gamma_{12}-\Gamma_{14} \\
\Gamma_{21}-\Gamma_{23} & \Gamma_{22}-\Gamma_{24}
\end{array}\right]=0
$$

For clamped-free beams, the boundary conditions are given by

$$
\begin{gathered}
Y_{1}(0)=0, \quad Y_{1}^{\prime}(0)=0, \\
(E I)_{n} Y_{n}^{\prime \prime}(L)=0, \quad\left((E I)_{n} Y_{n}^{\prime \prime}(L)\right)^{\prime}=0 .
\end{gathered}
$$

With the derivation similar to the above, using (44a), (44b), and (21), it can be obtained that

$$
\begin{gathered}
H_{1}+K_{1}=0, \quad J_{1}+L_{1}=0, \\
\boldsymbol{\Lambda}_{3} \mathbf{M}_{(n)}=\mathbf{0},
\end{gathered}
$$


TABLE 1: The material and physical parameters in [27].

\begin{tabular}{lcccc}
\hline$b(\mathrm{~mm})$ & $h(\mathrm{~mm})$ & $E_{1}(\mathrm{GPa})$ & $\nu_{1}$ & $\rho_{1}\left(\mathrm{~kg} / \mathrm{m}^{3}\right)$ \\
\hline 100 & 100 & 70 & 0.33 & 2780 \\
\hline
\end{tabular}

where

$$
\Lambda_{3}=\left[\begin{array}{cc}
-(E I)_{n} \varsigma_{n}^{2} \sin \varsigma_{n} l_{n} & -(E I)_{n} \varsigma_{n}^{3} \cos \varsigma_{n} l_{n} \\
-(E I)_{n} \varsigma_{n}^{2} \cos \varsigma_{n} l_{n} & (E I)_{n} \varsigma_{n}^{3} \sin \varsigma_{n} l_{n} \\
(E I)_{n} \varsigma_{n}^{2} \sinh \varsigma_{n} l_{n} & (E I)_{n} \varsigma_{n}^{3} \cosh \varsigma_{n} l_{n} \\
(E I)_{n} \varsigma_{n}^{2} \cosh \varsigma_{n} l_{n} & (E I)_{n} \varsigma_{n}^{3} \sinh \varsigma_{n} l_{n}
\end{array}\right]^{T} .
$$

In the same way, following matrix equations can be obtained as

$$
\begin{gathered}
\Lambda_{3} \mathbf{Z M}_{(1)}=\Gamma_{3} \mathbf{M}_{(1)}=\mathbf{0}, \\
\Gamma_{3}=\Lambda_{3} \mathbf{Z}, \\
\Gamma_{3} \mathbf{M}_{(1)}=\left[\begin{array}{llll}
\Gamma_{11} & \Gamma_{12} & \Gamma_{13} & \Gamma_{14} \\
\Gamma_{21} & \Gamma_{22} & \Gamma_{23} & \Gamma_{24}
\end{array}\right]\left[\begin{array}{c}
H_{1} \\
J_{1} \\
-H_{1} \\
-J_{1}
\end{array}\right]=\mathbf{0} .
\end{gathered}
$$

The characteristic equation of the clamped-free FGM beam can be obtained as

$$
\operatorname{det}\left[\begin{array}{ll}
\Gamma_{11}-\Gamma_{13} & \Gamma_{12}-\Gamma_{14} \\
\Gamma_{21}-\Gamma_{23} & \Gamma_{22}-\Gamma_{24}
\end{array}\right]=0
$$

It should be noted that the characteristic equations of beams under other arbitrary boundary conditions can also be determined using the derivation processes above.

\section{Validation of the Proposed Method}

At first, the convergence of the proposed method is discussed. Dividing a hinged-hinged beam of $E_{2} / E_{1}=1, c / L=0.5$, and $L / h=10$ into $n$ segments, the natural frequencies of the beam with an open edge crack of different depth are determined. The material and physical parameters of the beam in this case are consistent with those of Yang and Chen [27] and are listed in Table 1. Figure 2 shows the first three natural frequencies of the beam when different value of $n$ is adopted. The good convergence of the proposed method can be observed in Figure 2. The curves in the figure show that, for a beam with a crack of different depth, the frequency of each order reaches corresponding convergent solution when $n$ is larger than 64 .

To verify the validity of the method in this paper, comparisons of the results obtained from the present method with those from 3D FEM and available data in the literature are presented. A MATLAB code is written to achieve the theoretical solution in this paper, and the commercial software package ABAQUS is used to complete the 3D FE simulation. During 3D FE simulation, according to the fact that a FGM beam can be modeled as a multilayer beam when a sufficient
TABLE 2: The first three nondimensional natural frequencies of intact FGM beams.

\begin{tabular}{lcccc}
\hline$E_{2} / E_{1}=\rho_{2} / \rho_{1}$ & $\bar{\omega}_{1}$ & $\bar{\omega}_{2}$ & $\bar{\omega}_{3}$ \\
\hline Clamped-free & & & & \\
& Yang and Chen [27] & 0.83 & 5.18 & 14.49 \\
0.2 & Present & 0.8258 & 5.1752 & 14.4910 \\
& 3D FEM & 0.8269 & 5.1270 & 14.1144 \\
& Yang and Chen [27] & 0.88 & 5.51 & 15.42 \\
1.0 & Present & 0.8790 & 5.5086 & 15.4243 \\
& 3D FEM & 0.8799 & 5.4501 & 15.0078 \\
5.0 & Yang and Chen [27] & 0.83 & 5.18 & 14.49 \\
& Present & 0.8258 & 5.1752 & 14.4910 \\
& 3D FEM & 0.8268 & 5.1270 & 14.1144
\end{tabular}

Clamped-clamped

$$
\begin{array}{cccc}
\text { Yang and Chen [27] } & 2.51 & 9.27 & 21.07 \\
\text { Present } & 2.3181 & 9.2724 & 20.8628 \\
\text { 3D FEM } & 2.3085 & 9.1219 & 20.0568 \\
\text { Yang and Chen [27] } & 2.47 & 9.87 & 22.21 \\
\text { Present } & 2.4674 & 9.8696 & 22.2066 \\
\text { 3D FEM } & 2.4579 & 9.7116 & 21.4361
\end{array}
$$

$\begin{array}{llll}\text { Present } & 2.3181 & 9.2724 & 20.8628\end{array}$

$\begin{array}{llll}\text { Present } & 2.4674 & 9.8696 & 22.2066\end{array}$

$\begin{array}{ccccc}5.0 & \text { Yang and Chen [27] } & 2.51 & 9.27 & 21.07 \\ & \text { Present } & 2.3181 & 9.2724 & 20.8628\end{array}$

Hinged-hinged

$\begin{array}{llll}\text { 3D FEM } & 2.3085 & 9.1219 & 20.0568\end{array}$

\begin{tabular}{cccc} 
Yang and Chen [27] & 5.25 & 14.49 & 28.40 \\
Present & 5.2548 & 14.4852 & 28.3968 \\
3D FEM & 5.2050 & 14.0623 & 26.8927 \\
Yang and Chen [27] & 5.59 & 15.42 & 30.23 \\
Present & 5.5933 & 15.4182 & 30.2258 \\
3D FEM & 5.5319 & 14.9257 & 28.4977 \\
Yang and Chen [27] & 5.25 & 14.49 & 28.40 \\
Present & 5.2548 & 14.4852 & 28.3968 \\
3D FEM & 5.2050 & 14.0623 & 26.8927 \\
\hline
\end{tabular}

number of layers are adopted [31], 30-layer elements are divided along the thickness of the beam. Complete integral hexahedral 8-node elements are used in the element meshing, and the size of the elements adjacent to the crack is $3.33 \mathrm{~mm} \times$ $10 \mathrm{~mm} \times 10 \mathrm{~mm}$ and the size of the other elements is $3.33 \mathrm{~mm}$ $\times 16.66 \mathrm{~mm} \times 10 \mathrm{~mm}$. To model an open edge crack in the beam, elements at the location clinging to the left and right surfaces of the crack are just adjacent to each other and the coincident notes are not coupled, as shown in Figure 3. The material parameters are constant in each layer and the average values of the top and bottom surfaces of the corresponding layer.

The first three frequencies of intact FMG beams $(L / h=$ 10) with different material gradients are calculated by the present method and 3D FEM. The material and physical parameters of the FGM beams discussed in this case are those listed in Table 1. The frequencies of clamped-free, hinged-hinged, and clamped-clamped FGM beams determined herein and those of Yang and Chen [27] are listed in Table 2. All the results in the table are normalized by $\bar{\omega}_{i}=\omega_{i} / \sqrt{D_{0} / \bar{\rho}_{0} A}$, where $D_{0}$ and $\bar{\rho}_{0} A$ are the corresponding values of $D_{1}$ and $\bar{\rho} A$ of an isotropic homogeneous beam $\left(E_{2} / E_{1}=\rho_{2} / \rho_{1}=1.0\right)$. The comparison in Table 2 shows 

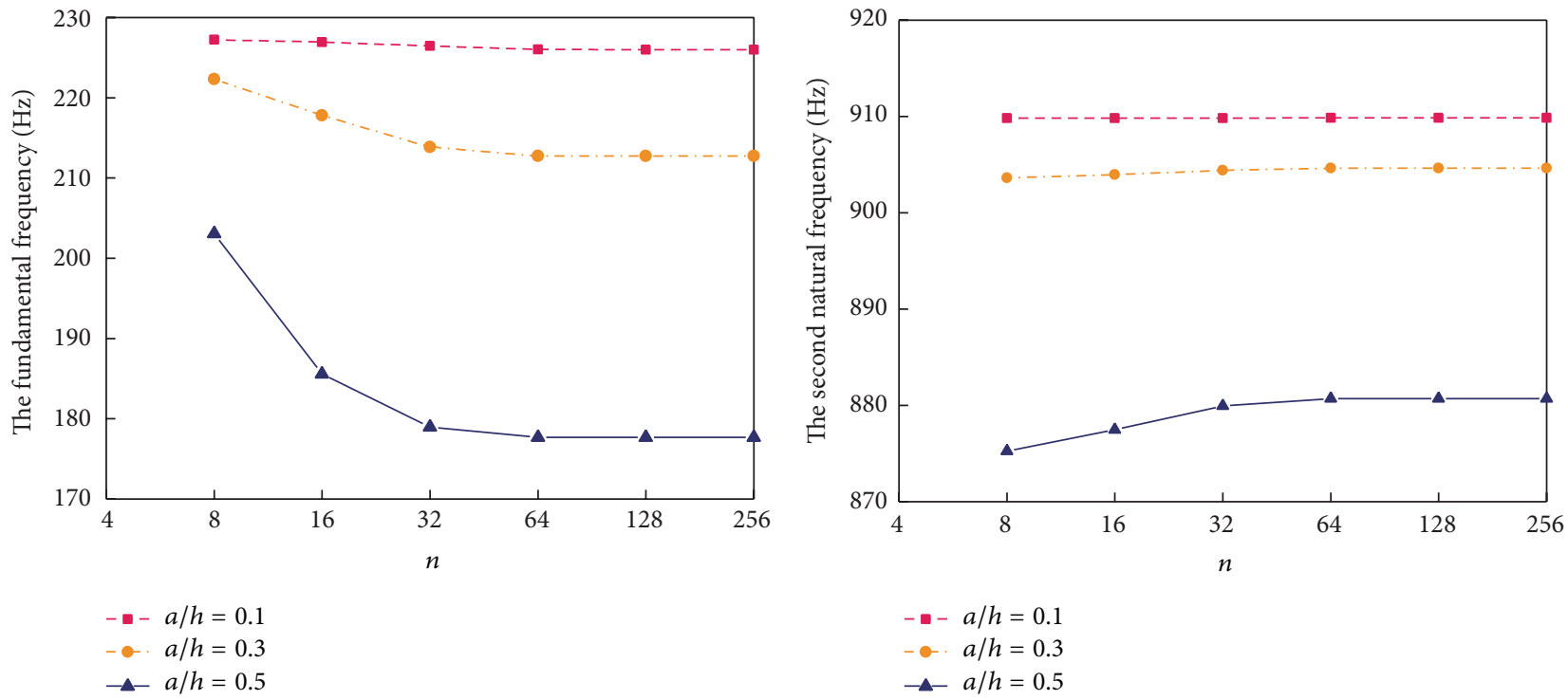

(a)

(b)

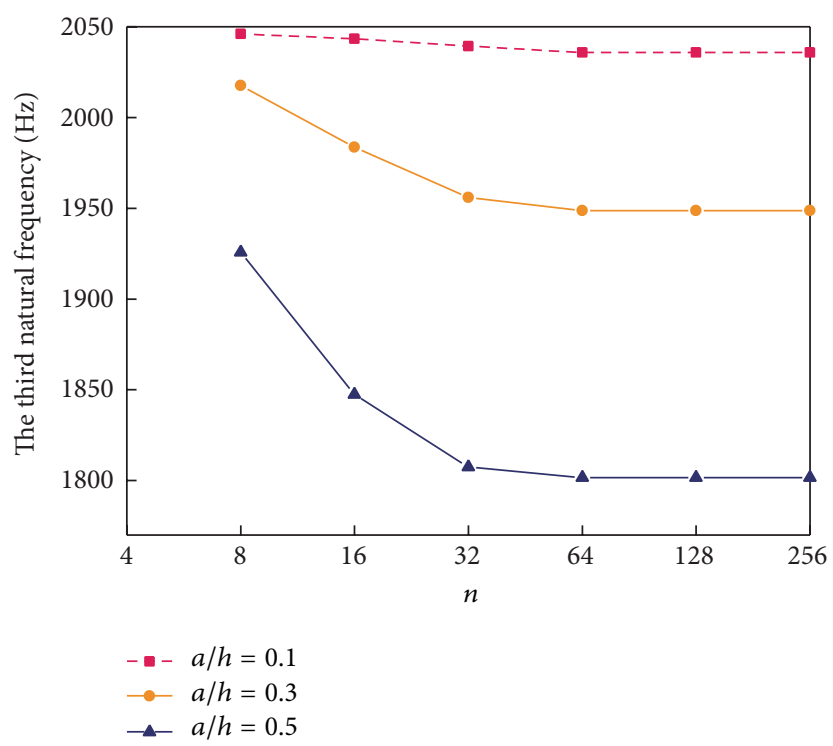

(c)

FiguRE 2: The first three natural frequencies of a hinged-hinged isotropic homogenous beam containing a single open edge crack: (a) the fundamental frequency, (b) the second frequency, and (c) the third frequency.

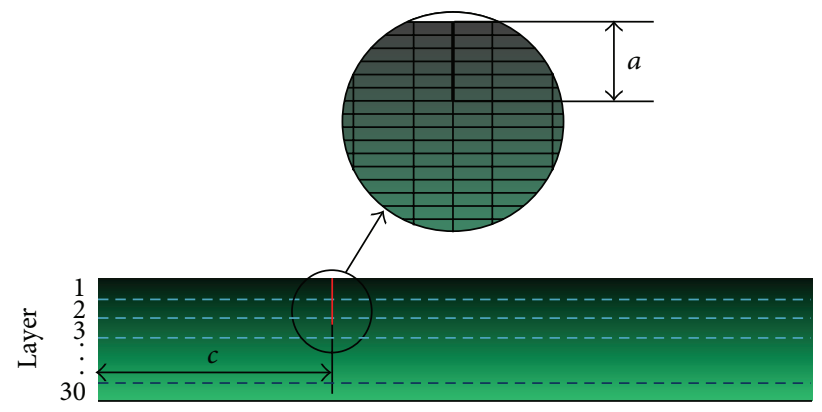

Figure 3: The 3D FE model of a cracked FGM beam. 


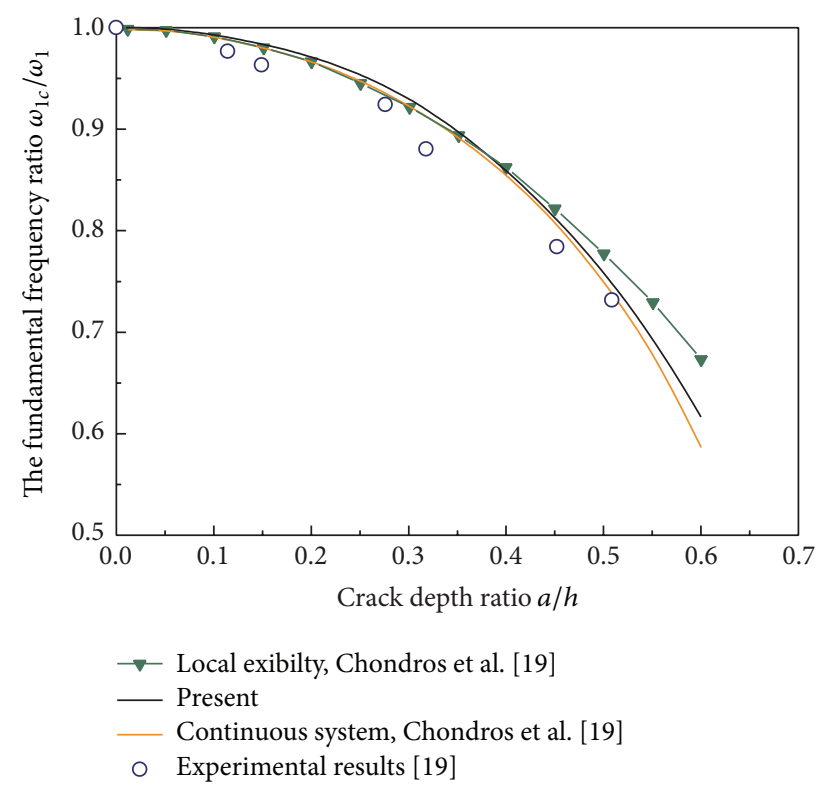

FIGURE 4: The fundamental frequency ratio of a cracked cantilever beam.

TABLE 3: The material and physical parameters in [19].

\begin{tabular}{lccccc}
\hline$L(\mathrm{~mm})$ & $b(\mathrm{~mm})$ & $h(\mathrm{~mm})$ & $E(\mathrm{GPa})$ & $\nu$ & $\rho\left(\mathrm{kg} / \mathrm{m}^{3}\right)$ \\
\hline 235 & 6 & 25.4 & 70 & 0.33 & 2800 \\
\hline
\end{tabular}

that the frequencies calculated by the present method agree well with the results of 3D FEM and those of Yang and Chen [27]. As stated in [27], with the identical valve of $\sqrt{D_{0} / \bar{\rho}_{0} A}$, the nondimensional natural frequencies of beams of $E_{2} / E_{1}=$ $\rho_{2} / \rho_{1}=0.2$ and $E_{2} / E_{1}=\rho_{2} / \rho_{1}=5.0$ are identical in Table 2 .

In another validation example, the first natural frequencies of isotropic homogeneous cantilever beams with a single crack at midspan are calculated. Figure 4 shows a comparison of the fundamental frequency ratios obtained by the present method and those of Chondros et al. [19]. The material and physical parameters are taken from [19] and are listed in Table 3. The fundamental frequency ratio $\omega_{1 c} / \omega_{1}$ denotes attenuation of the fundamental frequency for the cracked beam, $\omega_{1 c}$ is the fundamental frequency of the cracked beam, and $\omega_{1}$ is the fundamental frequency of the intact beam. Figure 4 shows that the present results correlate well with those theoretical results and experimental data of Chondros et al. [19] at different crack depths up to $a / h=0.6$.

By the proposed method and 3D FEM, the first two frequency ratios of a hinged-hinged FGM beam with an open edge crack at varying locations are determined. Figure 5 gives a comparison of the results herein and those in $[27,29]$. The slenderness ratio of the beams is $L / h=20$, the crack depth ratio is $a / h=0.2$, and the material and physical parameters are those in Table 1. Figure 5 confirms that the present results are highly similar to those of [29] and 3D FEM.

In the last verification example, the fundamental frequency of a FGM beam containing a single crack at the midpoint of the beam is calculated assuming that crack depth varies from $a / h=0$ to $a / h=0.5$. Young's modulus ratio is

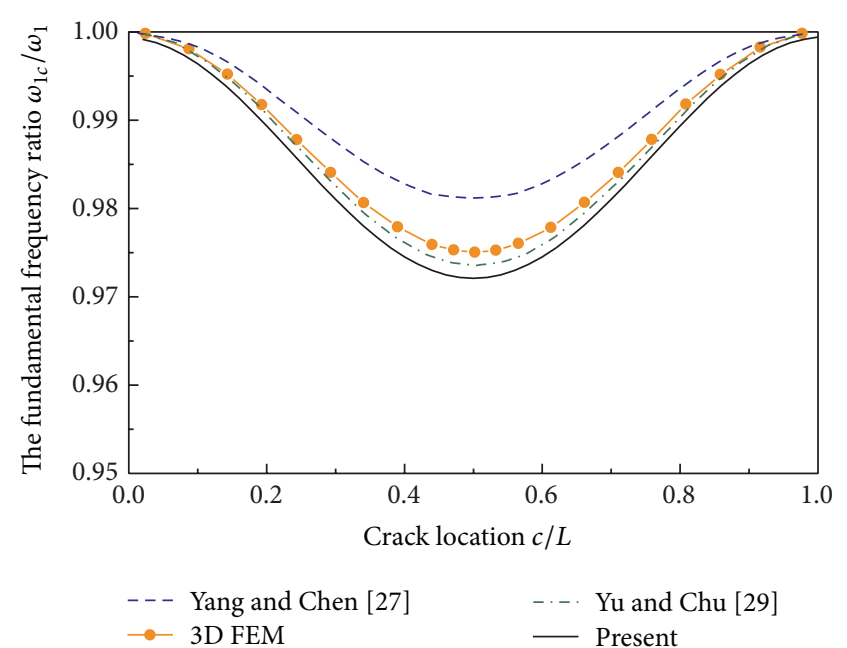

(a)

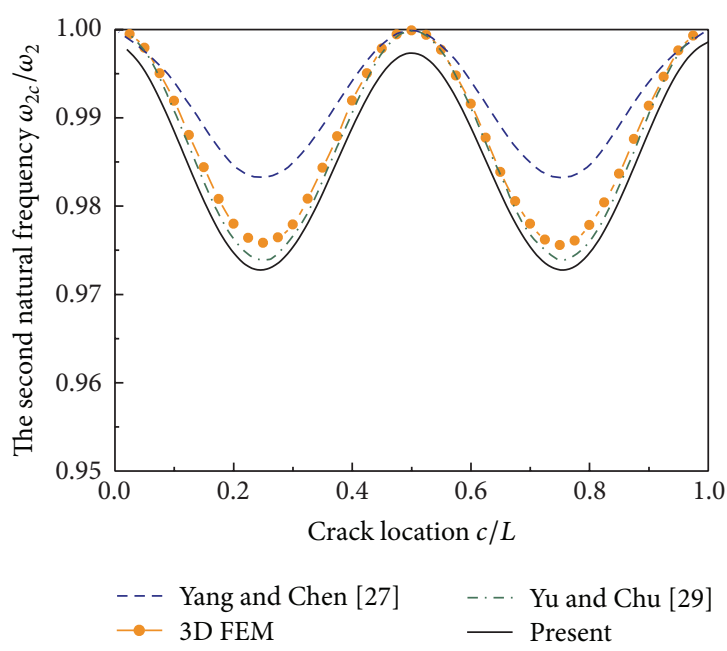

(b)

FIGURE 5: The first two frequency ratios of a hinged-hinged FGM beam with an open edge crack at varying locations: (a) the fundamental frequency ratio and (b) the second frequency ratio.

$E_{2} / E_{1}=0.2$ and the material and physical parameters are given by Table 1 . The fundamental frequency ratios $\omega_{1 c} / \omega_{1}$ obtained by the present method and by 3D FEM are listed in Table 4. It can be found that, for the FGM beams with different slenderness ratios and crack depths, the present results are extremely close to those obtained by 3D FEM. Due to the tiny precision decrease of the bending stiffness model for deeper cracks, the errors in Table 4 increase slightly with the increase of the crack depth. However, the errors remain at very low levels even in the case in which the crack depth ratio increases to $a / h=0.5$.

With the examples and the comparisons above the validity and accuracy of the present method are demonstrated.

\section{Numerical Results and Discussions}

After testing the proposed method, a series of vibration analysis of cracked FGM beams are carried out to discuss 

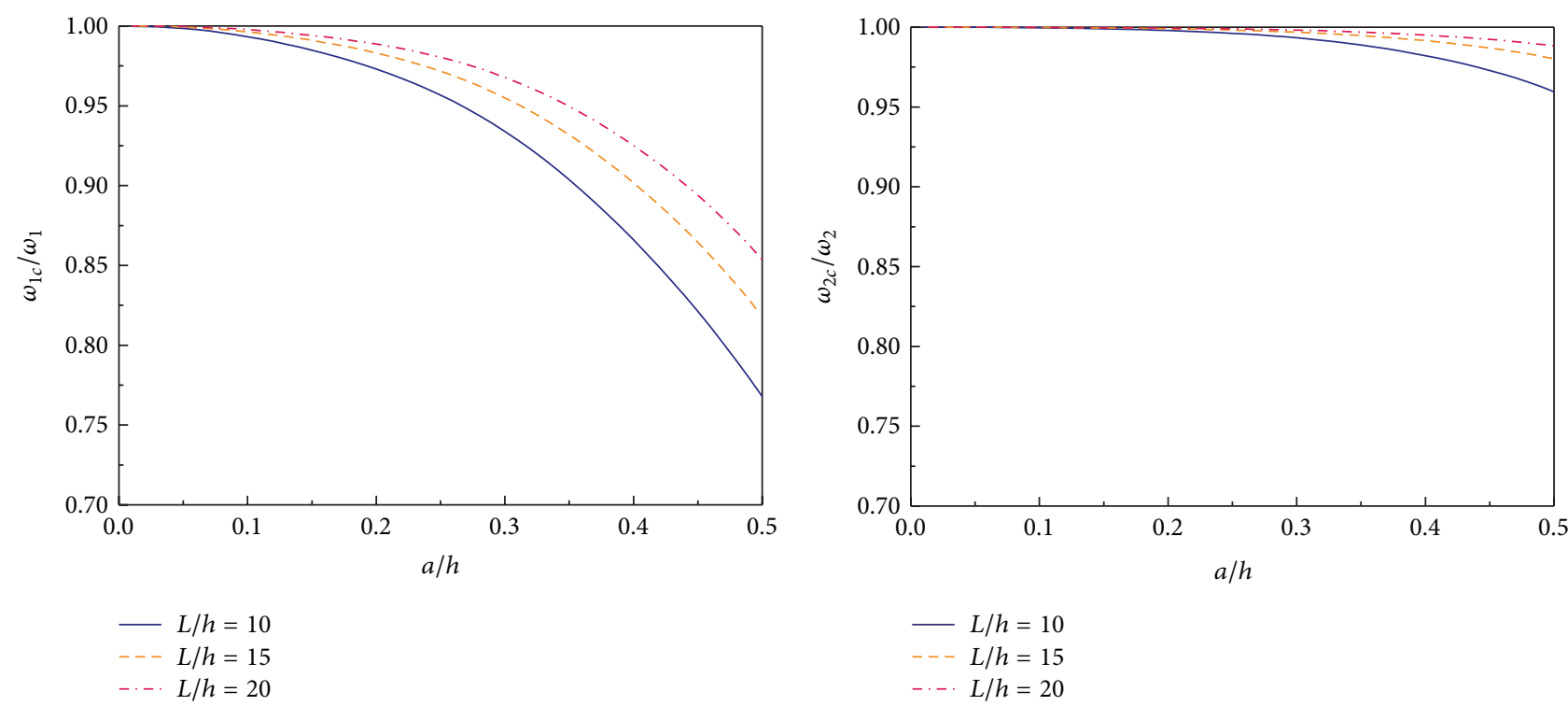

(a)

(b)

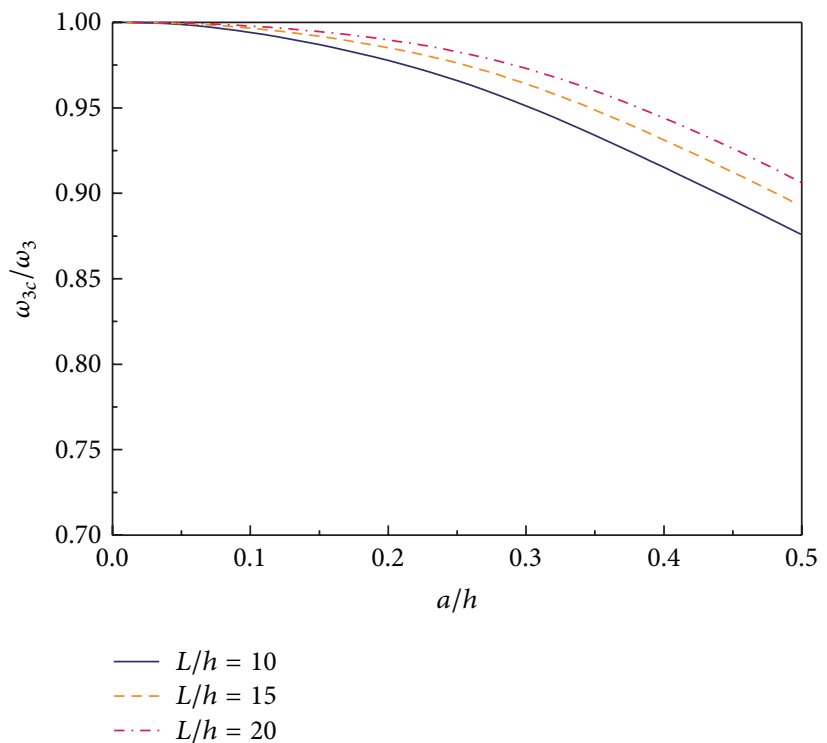

(c)

FIGURE 6: The first three frequency ratios of hinged-hinged isotropic homogenous beams with different slenderness ratios and containing a single open edge crack of varying depths: (a) the fundamental frequency ratio, (b) the second frequency ratio, and (c) the third frequency ratio.

TABLE 4: The fundamental frequency ratio of cracked FGM beams with different crack depth.

\begin{tabular}{lcccc}
\hline & \multicolumn{2}{c}{3 D FEM } & \multicolumn{2}{c}{ Present } \\
& $L / h=10$ & $L / h=20$ & $L / h=10$ & $L / h=20$ \\
& $\omega_{1 c} / \omega_{1}$ & $\omega_{1 c} / \omega_{1}$ & $\omega_{1 c} / \omega_{1}$ & $\omega_{1 c} / \omega_{1}$ \\
\hline$a / h=0.1$ & 0.9890 & 0.9943 & 0.9863 & 0.9934 \\
$a / h=0.2$ & 0.9545 & 0.9760 & 0.9501 & 0.9726 \\
$a / h=0.3$ & 0.8972 & 0.9434 & 0.8947 & 0.9376 \\
$a / h=0.4$ & 0.8162 & 0.8927 & 0.8087 & 0.8830 \\
$a / h=0.5$ & 0.7117 & 0.8084 & 0.7043 & 0.7994 \\
\hline
\end{tabular}

the influences of crack depth, crack location, distribution of material property, and slenderness ratio on the natural frequencies of the cracked FGM beams. The materials and physical parameters used in the following discussions are provided by Table 1 .

The plots of the first three natural frequency ratios of hinged-hinged cracked isotropic homogenous beams with different slenderness ratios are shown in Figure 6. The crack depth in this case increases gradually from $a / h=0$ to $a / h=$ 0.5 and the crack location remains constant at $c / L=0.5$. As expected, the first three frequency ratios decrease as the crack depth rate increases. The presence of cracks causes severer 

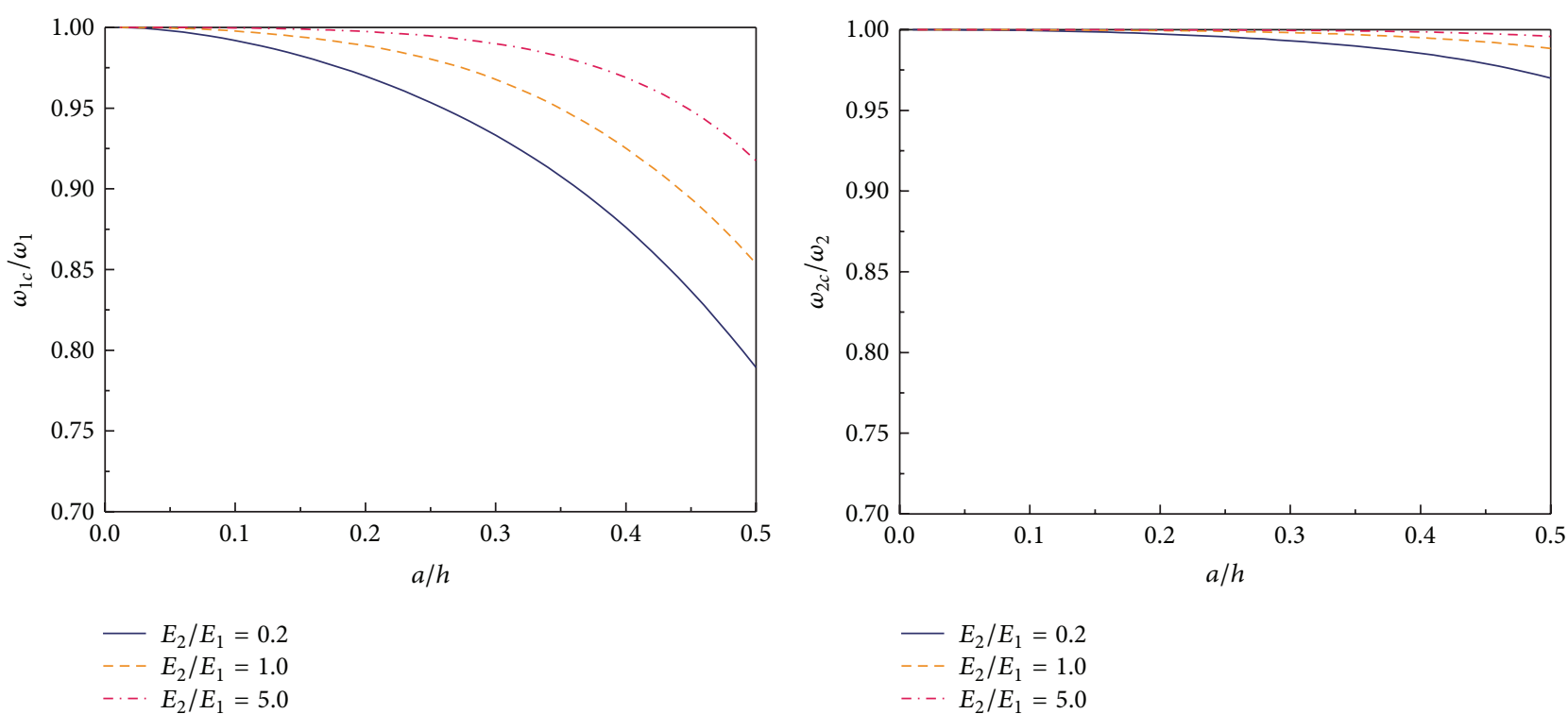

(a)

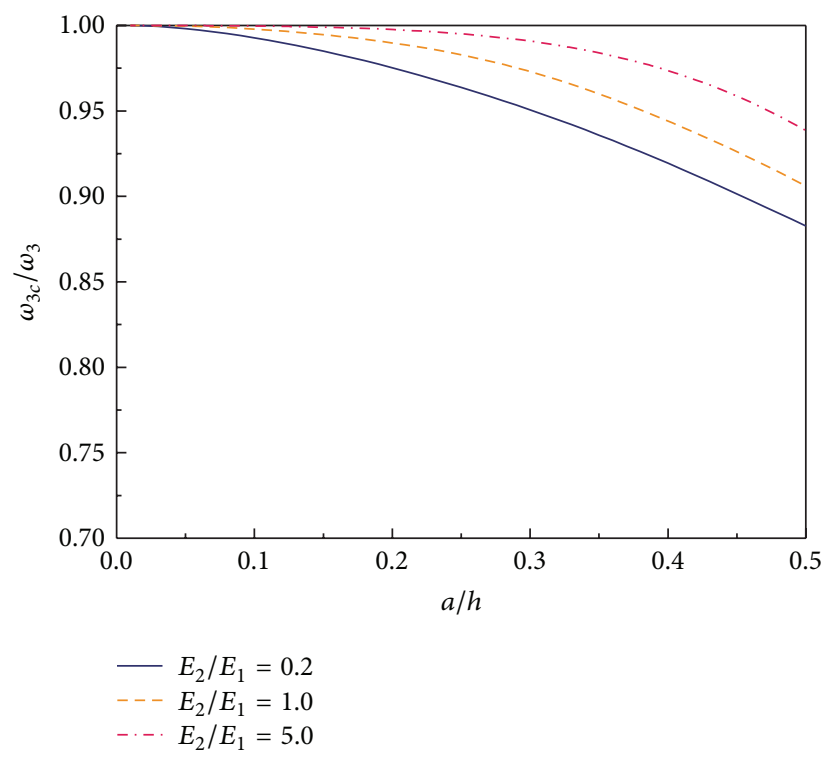

(c)

Figure 7: The first three frequency ratios of hinged-hinged FGM beams with different Young's modulus ratios and containing a single open edge crack of varying depths: (a) the fundamental frequency ratio, (b) the second frequency ratio, and (c) the third frequency ratio.

frequency attenuation for beams with smaller slenderness ratio, which indicates that the natural frequencies of beams with lower slenderness ratios are more sensitive to cracks. Figure 6 also shows that such a trend becomes more obvious for beams with deeper cracks.

Figure 7 shows the first three natural frequency ratios of cracked FGM beams with common slenderness ratio $L / h=$ 20 but different Young's modulus ratios $E_{2} / E_{1}$. The cracks are located at midpoints of the beams. For beams with lower Young's modulus ratios, lower frequency ratios of the FGM beams may be observed under the same crack depth. This observation indicates that FGM beams with lower Young's modulus ratios are more sensitive to the cracks. Figure 7 further shows that such a trend is fairly obvious even if the crack depth is very shallow $(a / h<0.1)$.

Figures 8 and 9 show the first three frequency ratio curves of cracked FGM beams with a common crack depth of $a / h=$ 0.3 and varying crack locations. Beams with the same Young's modulus ratio $E_{2} / E_{1}=0.2$ but different slenderness ratios are shown in Figure 8, while beams with common slenderness ratio $a / h=20$ but different Young's modulus ratios are shown in Figure 9. It is seen from Figures 8 and 9 that the natural frequency ratios vary with the normalized crack location. Maximum frequency attenuation can be observed at $c / L=$ $0.5, c / L=0.25$ and 0.75 , and $c / L=0.167$ and 0.833 for the first, second, and third mode, respectively. By contrast, 

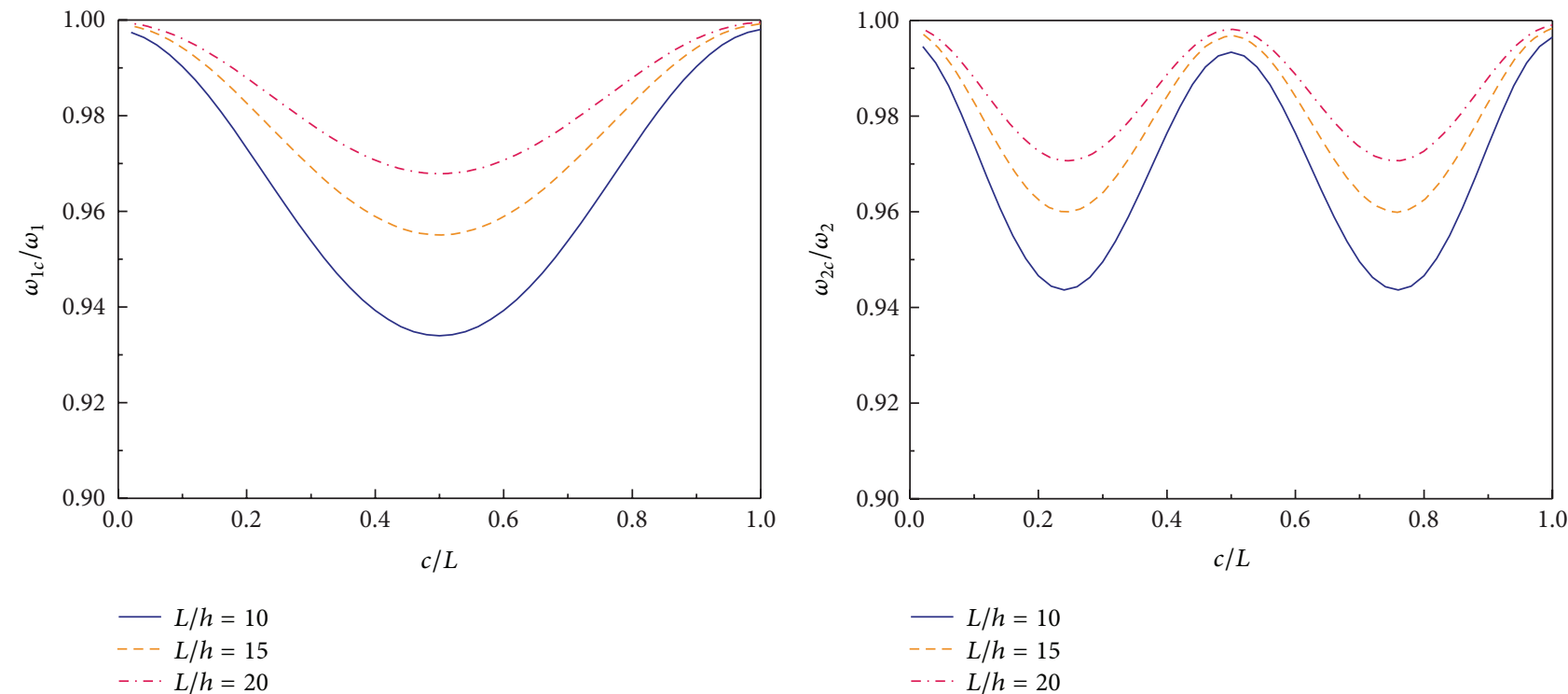

(a)

(b)

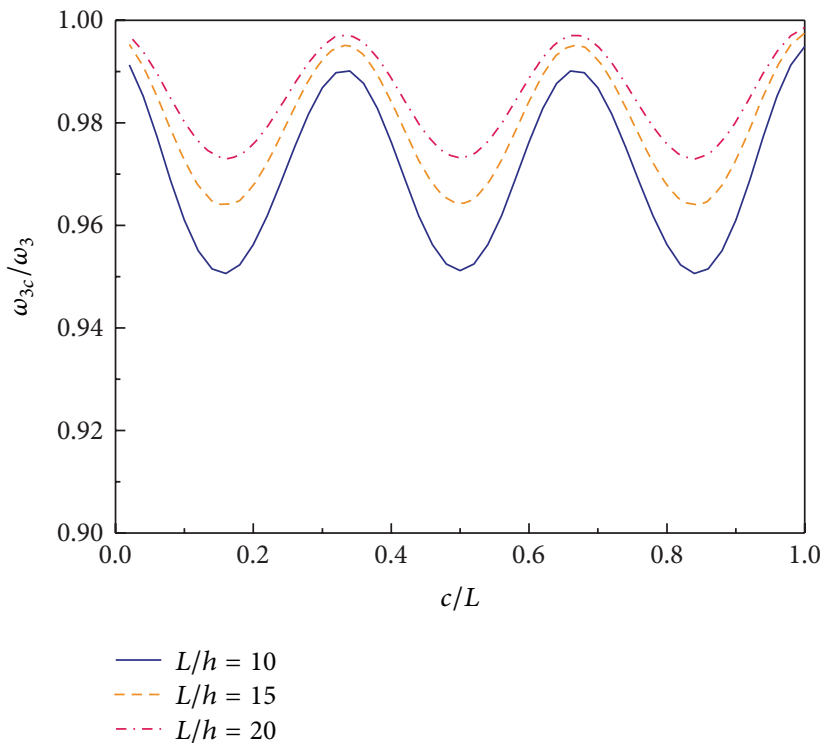

(c)

FIGURE 8: The first three frequency ratios of hinged-hinged FGM beams with different slenderness ratios and containing a single open edge crack $(a / h=0.3)$ at varying locations: (a) the fundamental frequency ratio, (b) the second frequency ratio, and (c) the third frequency ratio.

for the first three modes in turn, the frequency attenuation is not obvious when the crack is located at the ends, at $c / L=$ 0.5 and at $c / L=0.333$ and 0.667 . That means when cracks are located at nodal points of the mode shape, these cracks present very slight effects on natural frequencies; cracks lead to the most significant frequency attenuation when they are located at the position where maximum displacement of the mode shape occurs. Figures 8 and 9 further show that the effects of cracks on higher order frequencies of the hingedhinged FGM beams tend to decline. Moreover, it can be observed in Figure 9, when $E_{2} / E_{1}=5.0$ that the minimum frequency ratios of the first three orders in turn are 09899, 0.9904 , and 0.9908 , which contrasts the values of 0.9332 ,
0.9429 , and 0.9501 obtained at $E_{2} / E_{1}=0.2$. That means the narrowing amplitude of frequency attenuation in higher order frequencies tend to be more obvious for the FGM beams with lower Young's modulus ratio.

\section{Conclusions}

Free vibration of FGM beams with open edge cracks is investigated in this paper based on Euler-Bernoulli beam theory and the continuous beam model. Several examples are introduced to verify the validity of the methods proposed in this paper. The numerical discussion shows that FGM beams with more stubby shapes and lower Young's modulus 

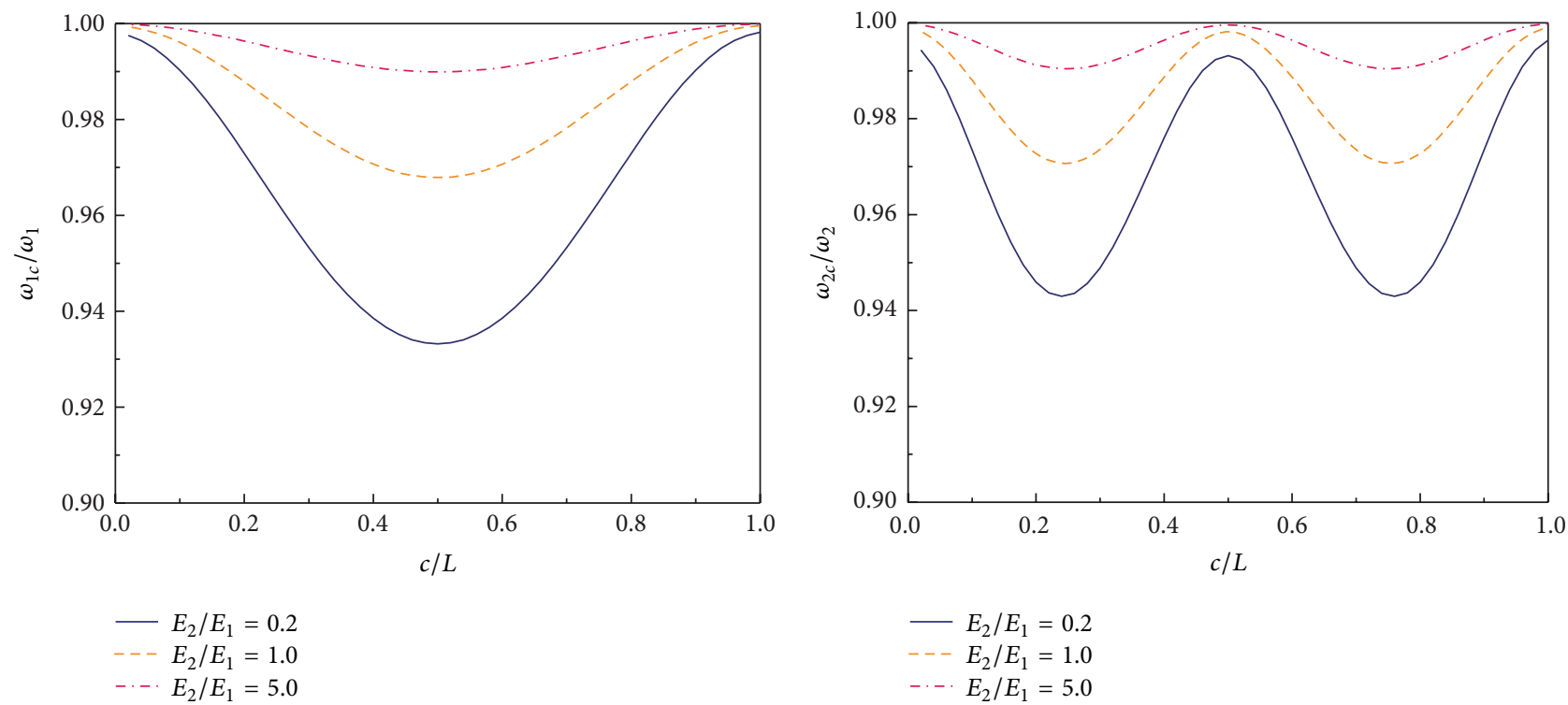

(a)

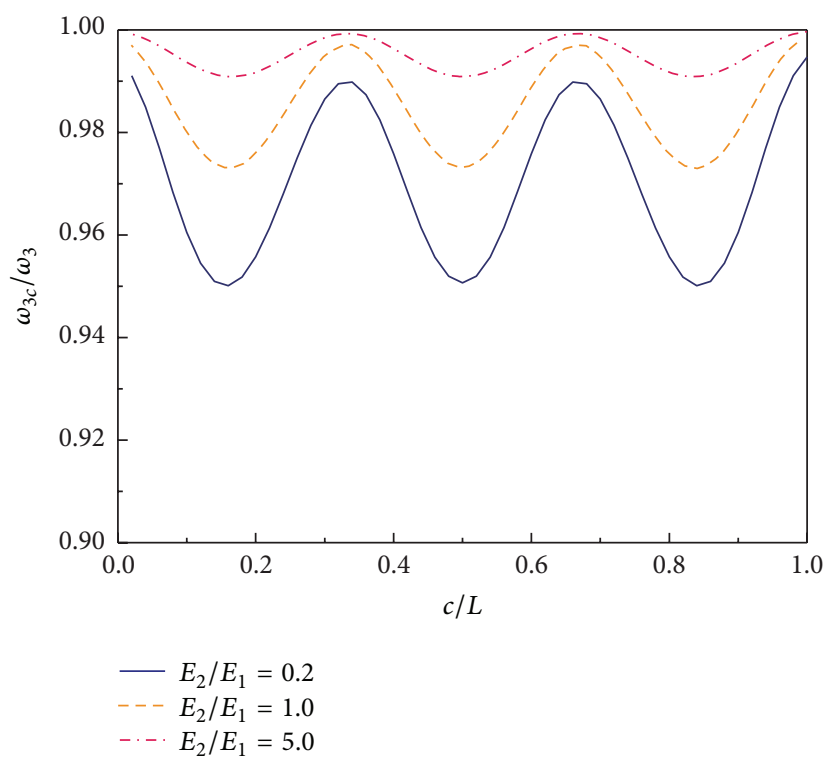

(c)

FIGURE 9: The first three frequency ratios of hinged-hinged FGM beams with different Young's modulus ratios and containing a single open edge crack $(a / h=0.3)$ at varying locations: (a) the fundamental frequency ratio, (b) the second frequency ratio, and (c) the third frequency ratio.

ratio are more sensitive to the presence of cracks. Moreover, if cracks are located at nodes of mode shapes, minimal effects on cracked FGM beams may be observed. Cracks lead to maximum effects if they are located at the position where maximum displacement of the mode shape occurs. The effects of cracks on the higher-order frequencies of hingedhinged FGM beams tend to decelerate, and such a trend becomes more obvious in FGM beams with lower Young's modulus ratio.

The main feature of this study is that, with the continuous stiffness model and the transform relation between FGM beams and isotropic homogeneous beams, the cracked FGM beam is treated as an intact beam with continuously varying bending stiffness along its longitudinal direction. Such an intact beam is divided into a sufficient number of segments and the transfer matrix from the first segment to the last segment is obtained based on the continuous conditions between interfacing segments. Then the characteristic equations and natural frequencies are determined for beams with given boundary conditions. Not limited to the discussions in this paper, the proposed method can be applied to cracked FGM beams with varying cross-sections and multiple cracks.

It should be noted that this study considers the cracked Euler-Bernoulli FGM beams containing open edge cracks only and the effects of damping are ignored. Therefore, the method proposed herein needs to be modified if it is to be 
applied to study short and thick FGM beams or in the cases in which the effects of damping are taken into account.

\section{Conflict of Interests}

The authors declare that there is no conflict of interests regarding the publication of this paper.

\section{Acknowledgments}

The work is financially supported by the National Natural Science Foundation of China (no. 11372257), by Sichuan Provincial Youth Science and Technology Innovation Team (no. 2013-TD-0004), and by the National Science Foundation for Young Scholars of China (no. 51405051).

\section{References}

[1] C. E. Rousseau and H. V. Tippur, "Influence of elastic gradient profiles on dynamically loaded functionally graded materials: cracks along the gradient," International Journal of Solids and Structures, vol. 38, no. 44-45, pp. 7839-7856, 2001.

[2] M. A. Benatta, A. Tounsi, I. Mechab, and M. B. Bachir, "Mathematical solution for bending of short hybrid composite beams with variable fibers spacing," Applied Mathematics and Computation, vol. 212, no. 2, pp. 337-348, 2009.

[3] H. J. Ding, D. J. Huang, and W. Q. Chen, "Elasticity solutions for plane anisotropic functionally graded beams," International Journal of Solids and Structures, vol. 44, no. 1, pp. 176-196, 2007.

[4] S. Ben-Oumrane, T. Abedlouahed, M. Ismail, B. B. Mohamed, M. Mustapha, and A. B. El Abbas, "A theoretical analysis of flexional bending of $\mathrm{Al} / \mathrm{Al}_{2} \mathrm{O}_{3}$ S-FGM thick beams," Computational Materials Science, vol. 44, no. 4, pp. 1344-1350, 2009.

[5] X. Y. Li, H. J. Ding, and W. Q. Chen, "Three-dimensional analytical solution for functionally graded magneto-electroelastic circular plates subjected to uniform load," Composite Structures, vol. 83, no. 4, pp. 381-390, 2008.

[6] X. Y. Li, H. J. Ding, and W. Q. Chen, "Elasticity solutions for a transversely isotropic functionally graded circular plate subject to an axisymmetric transverse load $q r^{k}$," International Journal of Solids and Structures, vol. 45, no. 1, pp. 191-210, 2008.

[7] S. R. Li and R. C. Batra, "Relations between buckling loads of functionally graded Timoshenko and homogeneous EulerBernoulli beams," Composite Structures, vol. 95, pp. 5-9, 2013.

[8] S. R. Li, D. F. Cao, and Z. Q. Wang, "Bending solutions of FGM Timoshenko beams from those of the homogenous EulerBernoulli beams," Applied Mathematical Modelling, vol. 37, no. 10-11, pp. 7077-7085, 2013.

[9] J. Arnaldo and J. Mazzei, "On the effect of functionally graded materials on resonances of rotating beams," Shock and Vibration, vol. 19, no. 4, pp. 707-718, 2012.

[10] A. D. Dimarogonas, Vibration Engineering, West Publishers, St. Paul, Minn, USA, 1976.

[11] N. Anifantis and A. D. Dimarogonas, "Stability of columns with a single crack subjected to follower and vertical loads," International Journal of Solids and Structures, vol. 19, no. 4, pp. 281-291, 1983.

[12] C. A. Papadopoulos and A. D. Dimarogonas, "Coupling of bending and torsional vibration of a cracked Timoshenko shaft," Ingenieur-Archiv, vol. 57, no. 4, pp. 257-266, 1987.
[13] J. W. Xiang, X. F. Chen, Q. Y. Mo, and Z. J. He, "Identification of crack in a rotor system based on wavelet finite element method," Finite Elements in Analysis and Design, vol. 43, no. 14, pp. 10681081, 2007.

[14] J. W. Xiang, Y. T. Zhong, X. F. Chen, and Z. J. He, "Crack detection in a shaft by combination of wavelet-based elements and genetic algorithm," International Journal of Solids and Structures, vol. 45, no. 17, pp. 4782-4795, 2008.

[15] X. Y. Li, X. Zhao, and Y. H. Li, "Green's functions of the forced vibration of Timoshenko beams with damping effect," Journal of Sound and Vibration, vol. 333, no. 6, pp. 1781-1795, 2014.

[16] S. Christides and A. D. S. Barr, "One-dimensional theory of cracked Bernoulli-Euler beams," International Journal of Mechanical Sciences, vol. 26, no. 11-12, pp. 639-648, 1984.

[17] S. Christides and A. D. S. Barr, "Torsional vibration of cracked beams of non-circular cross-section," International Journal of Mechanical Sciences, vol. 28, no. 7, pp. 473-490, 1986.

[18] M. H. H. Shen and C. Pierre, "Natural modes of Bernoulli-Euler beams with symmetric cracks," Journal of Sound and Vibration, vol. 138, no. 1, pp. 115-134, 1990.

[19] T. G. Chondros, A. D. Dimarogonas, and J. Yao, "A continuous cracked beam vibration theory," Journal of Sound and Vibration, vol. 215, no. 1, pp. 17-34, 1998.

[20] A. S. J. Swamidas, X. Yang, and R. Seshadri, "Identification of cracking in beam structures using Timoshenko and Euler formulations," Journal of Engineering Mechanics, vol. 130, no. 11, pp. 1297-1308, 2004.

[21] B. Chomette, A. Fernandes, and J. J. Sinou, "Cracks detection using active modal damping and piezoelectric components," Shock and Vibration, vol. 20, no. 4, pp. 619-631, 2013.

[22] F. Delale and F. Erdogan, "The crack problem for a nonhomogeneous plane," Journal of Applied Mechanics, vol. 50, no. 3, pp. 609-614, 1983.

[23] F. Delale and F. Erdogan, "Interface crack in a nonhomogeneous elastic medium," International Journal of Engineering Science, vol. 26, no. 6, pp. 559-568, 1988.

[24] M. Ozturk and F. Erdogan, "The axis symmetric crack problem in a nonhomogeneous medium," Journal of Applied Mechanics, vol. 60, no. 2, pp. 406-413, 1993.

[25] F. Erdogan and B. H. Wu, "The surface crack problem for a plate with functionally graded properties," Journal of Applied Mechanics, vol. 64, no. 3, pp. 449-456, 1997.

[26] Y. S. Chan, G. H. Paulino, and A. C. Fannjiang, "The crack problem for nonhomogneous materials under antiplane shear loading-a displacement based formulation," International Journal of Solids and Structures, vol. 38, no. 17, pp. 2980-3005, 2001.

[27] J. Yang and Y. Chen, "Free vibration and buckling analyses of functionally graded beams with edge cracks," Composite Structures, vol. 83, no. 1, pp. 48-60, 2008.

[28] L.-L. Ke, J. Yang, S. Kitipornchai, and Y. Xiang, "Flexural vibration and elastic buckling of a cracked timoshenko beam made of functionally graded materials," Mechanics of Advanced Materials and Structures, vol. 16, no. 6, pp. 488-502, 2009.

[29] Z. Yu and F. Chu, "Identification of crack in functionally graded material beams using the p-version of finite element method," Journal of Sound and Vibration, vol. 325, no. 1-2, pp. 69-84, 2009.

[30] K. Aydin, "Free vibration of functionally graded beams with arbitrary number of surface cracks," European Journal of Mechanics, A: Solids, vol. 42, pp. 112-124, 2013.

[31] Z. Zhong and Z. Cheng, "Fracture analysis of a functionally graded strip with arbitrary distributed material properties," International Journal of Solids and Structures, vol. 45, no. 13, pp. 3711-3725, 2008 

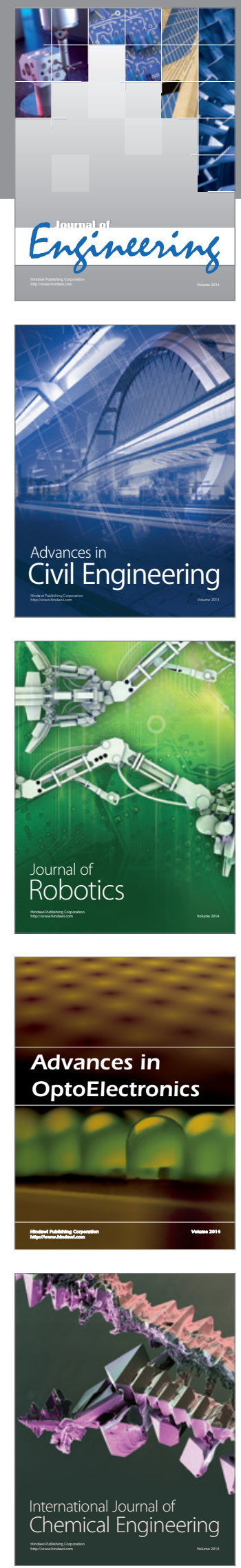

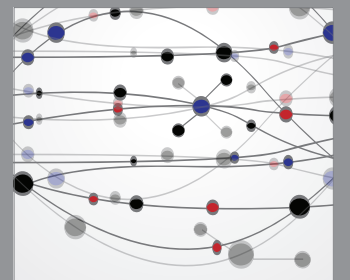

The Scientific World Journal
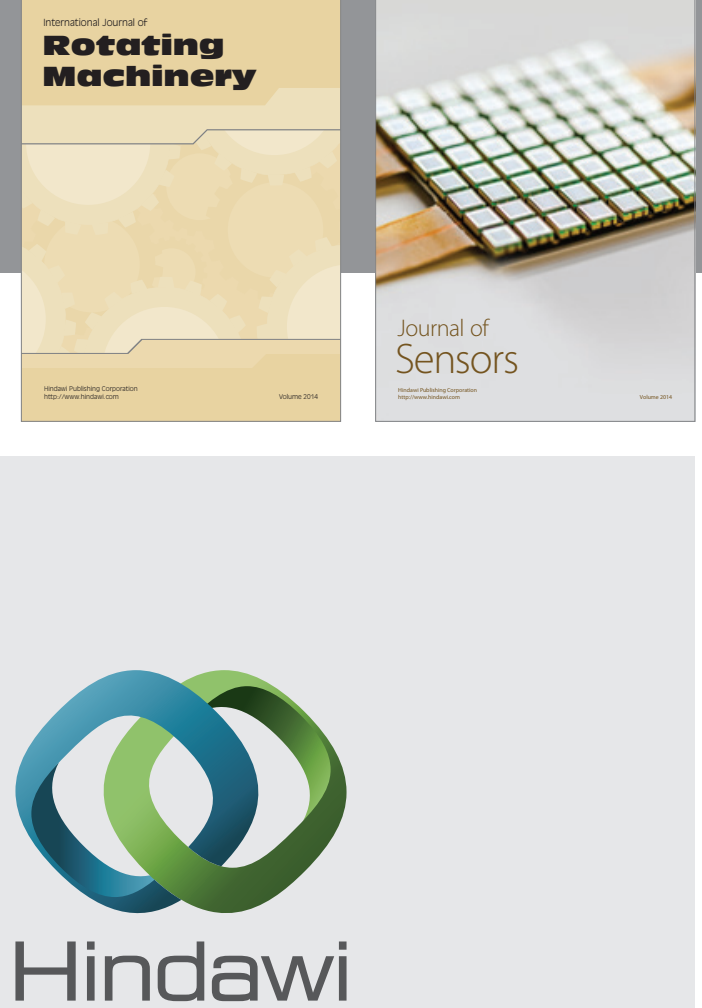

Submit your manuscripts at http://www.hindawi.com
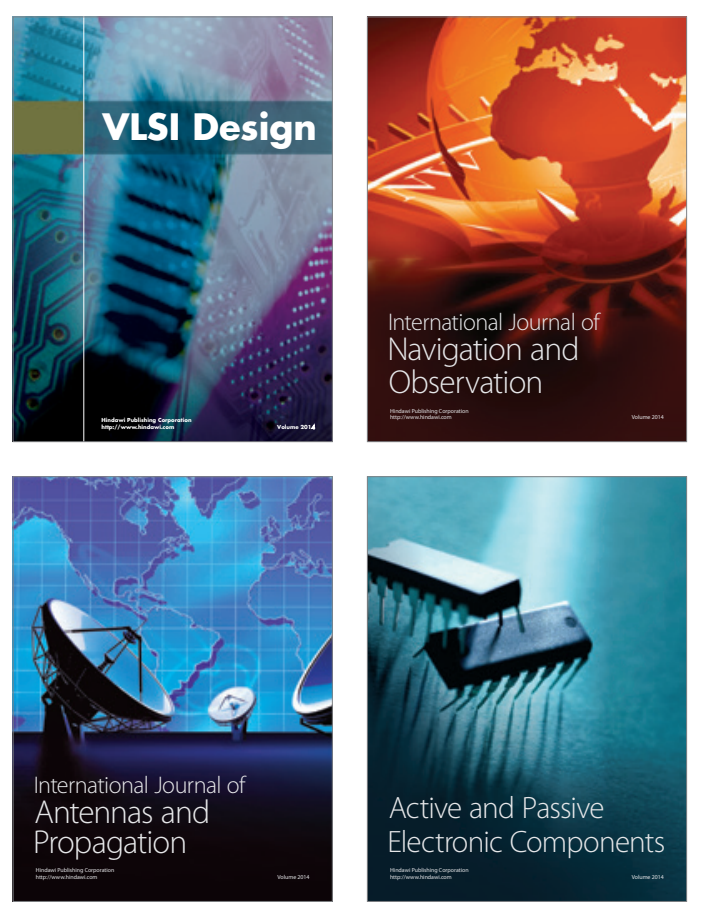
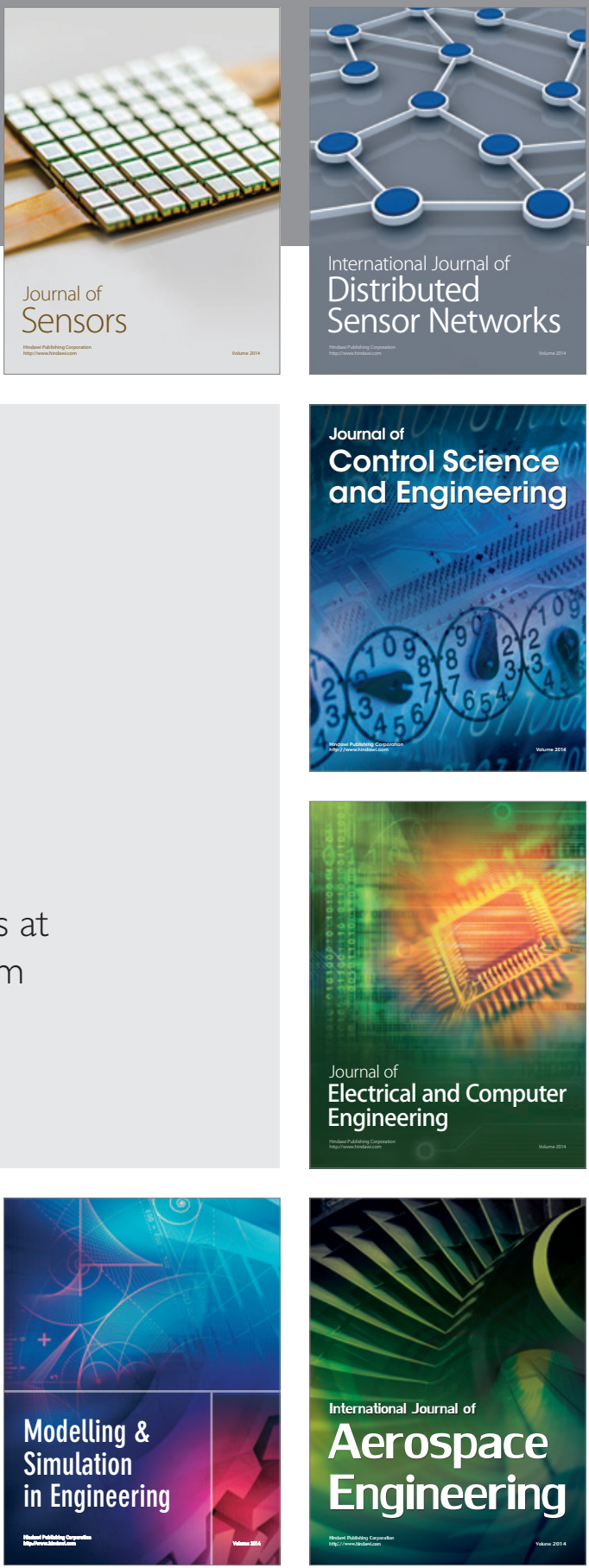

Journal of

Control Science

and Engineering
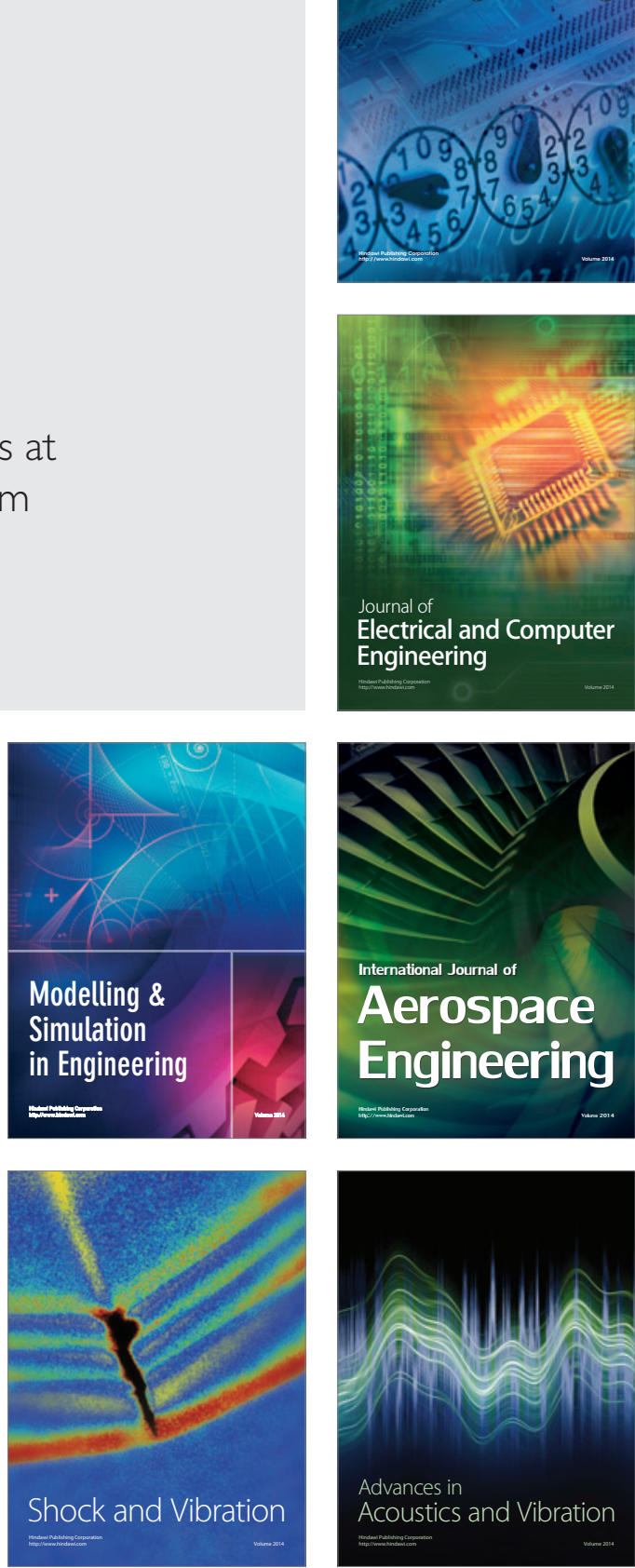